\title{
Induction of Tachykinin Gene and Peptide Expression in Guinea Pig Nodose Primary Afferent Neurons by Allergic Airway Inflammation
}

\author{
Axel Fischer, ${ }^{\star}$ Gerard P. McGregor, ${ }^{\ddagger}$ Alois Saria, ${ }^{\S}$ Bärbel Philippin, ${ }^{\star}$ and Wolfgang Kummer \\ *Institute for Anatomy and Cell Biology, ${ }^{\ddagger}$ Institute for Physiology, Philipps University, Marburg, Germany; ${ }^{\circledR}$ Neurochemical Unit,

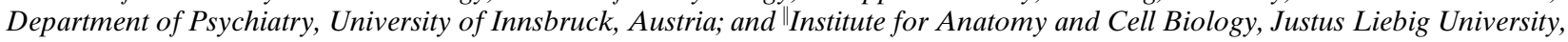 \\ Giessen, Germany
}

\begin{abstract}
Substance P (SP), neurokinin A (NKA), and calcitonin gene-related peptide (CGRP) have potent proinflammatory effects in the airways. They are released from sensory nerve endings originating in jugular and dorsal root ganglia. However, the major sensory supply to the airways originates from the nodose ganglion. In this study, we evaluated changes in neuropeptide biosynthesis in the sensory airway innervation of ovalbumin-sensitized and -challenged guinea pigs at the mRNA and peptide level. In the airways, a threeto fourfold increase of SP, NKA, and CGRP, was seen $24 \mathrm{~h}$ following allergen challenge. Whereas no evidence of local tachykinin biosynthesis was found $12 \mathrm{~h}$ after challenge, increased levels of preprotachykinin (PPT)-A mRNA (encoding SP and NKA) were found in nodose ganglia. Quantitative in situ hybridization indicated that this increase could be accounted for by de novo induction of PPT-A mRNA in nodose ganglion neurons. Quantitative immunohistochemistry showed that $24 \mathrm{~h}$ after challenge, the number of tachykinin-immunoreactive nodose ganglion neurons had increased by $25 \%$. Their projection to the airways was shown. Changes in other sensory ganglia innervating the airways were not evident. These findings suggest that an induction of sensory neuropeptides in nodose ganglion neurons is crucially involved in the increase of airway hyperreactivity in the late response to allergen challenge. (J. Clin. Invest. 1996. 98:2284-2291.) Key words: neurokinin A • ovalbumin sensitization • NANC-innervation • sensory innervation
\end{abstract}

\section{Introduction}

Allergic disorders of the respiratory tract, such as bronchial asthma, are considered chronic inflammatory diseases. Pharmacological studies and experiments using electrical field stimulation, have demonstrated that many of the proinflammatory processes involved in asthma, such as bronchoconstriction, mucus hypersecretion, and plasma extravasation are mimicked by neuropeptides $(1,2)$, that can be released from primary afferent (sensory) nerve endings by various stimuli (3).

Address correspondence to Dr. Axel Fischer, Institute for Anatomy and Cell Biology, Justus Liebig University, Aulweg 123, 35385 Giessen, Germany. Phone: 49-641-702-3940; FAX: 49-641-702-3977; E-mail: axel.fischer@anatomie.med.uni-giessen.de

Received for publication 9 February 1996 and accepted in revised form 23 September 1996.

J. Clin. Invest.

(C) The American Society for Clinical Investigation, Inc. 0021-9738/96/11/2284/08 \$2.00

Volume 98, Number 10, November 1996, 2284-2291
It appears that the predominant sensory neuropeptides are calcitonin gene-related peptide $(\mathrm{CGRP})^{1}$ and the tachykinins, substance P, and neurokinin A (4). Whereas it seems difficult to define their function under physiological conditions, a pathophysiological function for these neuropeptides as mediators of neurogenic inflammation has been well characterized (5), and has been postulated for the airways (6). Indeed, there is evidence to support the hypothesis of an involvement of endogenous tachykinins in allergic airway disease. For example, increased levels of tachykinins were measured in bronchoalveolar lavage fluids of asthmatic patients (7). In guinea pigs, release of endogenous sensory neuropeptides was shown to enhance nonspecific airway hyperresponsiveness (8).

Substance P (SP) and neurokinin A (NKA), which are derived from the same gene, the preprotachykinin (PPT) A gene, share a common $\mathrm{COOH}$-terminal amino acid sequence, which is responsible for most of their biological effects (9). SP and NKA can act through the same receptors but differ in their relative potency with respect to the tachykinin receptor subtypes. Within the airways, there is a differential distribution of these tachykinin receptor subtypes that may underly differences in the contribution of SP and NKA to the mediation of neurogenic inflammation (10). Thus, in the guinea pig, SP, acting through NK-1 receptors, may be more relevant than NKA for the mediation of extravasation and mucous secretion $(11,12)$, whereas NKA, acting through NK-2 receptors is an important mediator of bronchoconstriction (13). Pharmacological evidence suggests that CGRP, which is produced by the same neurons that produce SP and NKA, is the most relevant of these neuropeptides with regard to the mediation of vasodilatation (14).

In the airways, tachykinin-immunoreactive nerve fibers have been localized to the airway smooth muscle, submucosal glands, blood vessels of the lamina propria, and to nerve fibers innervating intrinsic neurons (15). As revealed by studies using retrograde neuronal tracing in combination with immunohistochemistry, in rat (16) and in guinea pig (17), nerve fibers innervating the trachea almost exclusively originate from the superior (jugular) and inferior (nodose) vagal sensory ganglion, whereas the lung also receives a sensory innervation originating from dorsal root ganglia. Interestingly, tachykinin immunoreactivity was not seen in nodose sensory neurons projecting to the airways, although they are the major source of the sensory airway innervation (17). Thus, tachykinin-immunoreactive nerve fibers innervating the airways originate from the jugular ganglion and from dorsal root ganglia $(17,18)$.

1. Abbreviations used in this paper: CGRP, calcitonin gene-related peptide; NK, neurokinin; NKA, neurokinin A; PB, phosphate buffer; PPT, preprotachykinin; SP, substance P. 
Activation or damage of neurons can lead to changes in neuropeptide biosynthesis that may include changes in the level of neuropeptide biosynthesis as well as induction or repression of neuropeptide gene expression (19). For example, in specific models of acute and chronic inflammation, plasticity of neuropeptide expression in dorsal root ganglion sensory neurons has been demonstrated $(20,21)$. So far, changes in the neuropeptide biosynthesis by sensory neurons in allergic inflammation have not been reported.

In the present study, we used the model of the actively sensitized and allergen- challenged guinea pig, which has been well characterized and shown to have many similarities to asthma of human airways (22). In this model, we investigated possible quantitative and qualitative changes in the neuropeptide biosynthesis of primary afferent neurons at the mRNA level, using northern blot analysis and in situ hybridization and at the neuropeptide level, using radioimmunoassay and by immunohistochemistry combined with retrograde neuronal tracing.

\section{Methods}

Active sensitization of guinea pigs to ovalbumin. The sensitization protocol was adapted from Sanjar et al. (22). Female specific pathogenfree guinea pigs of 200-250 g body weight (Charles River Wiga, Kieslegg, Germany) received intraperitoneal injections of ovalbumin (10 mg; Sigma GmbH, Munich, Germany), pertussis vaccine $(250 \mathrm{ml}$; Berna GmbH, Berne, Switzerland) and aluminium hydroxide (50 mg; Sigma) dissolved to a final volume of $1 \mathrm{ml}$ at day 1,14 , and 28. Control animals received the same injections except for the omission of ovalbumin. At day 35 all animals were tested by an intradermal injection of $50-\mathrm{ml}$ ovalbumin solution $(0.01 \% \mathrm{wt} / \mathrm{vol}$ in physiological saline) into the dorsal skin. Sensitization was considered successful when a doubling of skin thickness and an obvious allergic flare reaction were observed. Successfully sensitized and control animals were then exposed to the allergen by nebulization of $10-\mathrm{ml}$ ovalbumin solution $(0.1 \%)$ into a 4 liter chamber over 60 min using a pressure nebulizer (pari-boy type 37.00, pari-Werk GmbH, Starnberg, Germany). At each of the following time points and for each of the following techniques, five sensitized and five control animals were killed and tissues were removed: before, $0.5,1,3$, and $7 \mathrm{~d}$ after allergen challenge.

Scanning electron microscopy. Thoracic trachea and main stem bronchi of sensitized and control animals were removed $3 \mathrm{~d}$ after challenge and immersed in Zamboni's fixative ( $2 \%$ buffered formaldehyde, $15 \%$ saturated picric acid) for $12 \mathrm{~h}$, rinsed in $0.1 \mathrm{M}$ phosphate buffer (PB), and dehydrated in ascending ethanol concentrations. The anterior wall of the airways was cut and tissues were criticalpoint dried and sputtered with gold. Specimens were viewed with a Philips scanning electron microscope.

Northern blot analysis. Total RNA was extracted from pooled snap frozen sensory ganglia ( $n=4$ for each extraction) with guanidinium thiocyanate (GTC; Sigma Chemical Co., St. Louis, MO) followed by ultracentrifugation in cesium trifluoroacetate (CsTFA; Pharmacia, Freiburg, Germany) according to a modified protocol of Chirgwin et al. (23).

RNA-electrophoresis and -transfer to nylon filters (Hybond N; Amersham, Braunschweig, Germany) as well as the preparation of radiolabeled PPT-A and Actin- cDNAs and the subsequent prehybridization and hybridization steps, were performed as described before (24). In brief, the $\beta$-PPT-A cDNA insert, excised from the plasmid pG1-PPT (kindly provided by Dr. J.E. Krause, St. Louis, MO, 25), and the mouse Actin cDNA insert, excised from the plasmid AL.41 (Bluescript; Stratagene, Heidelberg, Germany), were radiolabeled using $\left[{ }^{32} \mathrm{P}\right] \mathrm{dCTP}$ (Amersham). For direct quantification, hybridized northern blots were measured using the "PhosphorImager" system (Molecular Dynamics Inc., Krefeld, Germany) and, for documentation, exposed to x-ray film (X-OMAT; Eastman Kodak Co., Rochester, NY).

In situ hybridization. Digoxigenin-labeled cRNA probes were obtained by in vitro transcription of the $\beta$-PPT-A cDNA using a nonradioactive nucleic acid detection kit (Boehringer Mannheim, Mannheim, Germany). In situ hybridization was performed on sections of paraformaldehyde-fixed (4\%, immersion fixation) ganglia and lung tissue according to a protocol described earlier (26).

For microscopic evaluation, slides with sections of the sensory ganglia were coded, to ensure that the investigator could not identify the tissues with respect to sensitization status and time point of tissue removal. Two sections were chosen at random, and in each section 100 neurons were examined for their PPT-A mRNA expression.

Double-labeling immunohistochemistry. Zamboni-fixed sensory ganglia (nodose and jugular ganglia, dorsal root ganglia T1-T6) and lung samples were rinsed in 0.1-M PB and cryoprotected with $18 \%$ sucrose in 0.1-M PB overnight. Sections of the lung and serial sections of the sensory ganglia were cut on a cryostat (model 500 OM Microm; Walldorf, Germany) at $12 \mathrm{~mm}$ and air dried for $30 \mathrm{~min}$. Two randomly chosen slides from the sensory ganglion series and lung sections were incubated with a blocking solution containing $1 \%$ BSA and $10 \%$ normal swine serum in $0.1-\mathrm{M}$ PB for $60 \mathrm{~min}$, followed by a combination of a monoclonal antibody from rat that recognizes both SP and NKA (Dunn, Asbach, Germany; dilution 1:200) and a polyclonal antiserum to CGRP from rabbit (Peninsula Laboratories Inc., Belmont, CA; dilution 1:800) overnight. After washing in PBS, a biotinylated anti-rat-immunoglobulin (Ig) from sheep (Amersham; dilution 1:50) was applied for $1 \mathrm{~h}$, and, after several washes in PBS, followed by a mixture of a streptavidin-Texas-Red-conjugate (Amersham; dilution 1:50) and an FITC-conjugated anti-rabbit-Ig from sheep (Wellcome Industries, Beckenham, UK; dilution 1:120) for $1 \mathrm{~h}$. Slides were washed thoroughly in PBS and placed on coverslips with buffered glycerol ( $\mathrm{pH}$ 8.6).

For microscopic evaluation with an epifluorescence microscope (model Axioplan; Zeiss, Oberkochen, Germany), sections were examined for their SP/NKA- and CGRP-immunoreactivity as described for in situ hybridization.

Retrograde neuronal tracing. At day 29 of the sensitization protocol, three sensitized and three control animals were anaesthetized by intramuscular injections of ketamine hydrochloride (Ketanest ${ }^{\mathrm{R}}$; Parke-Davis, Freiburg, Germany; $50 \mathrm{mg} / \mathrm{kg}$ ) followed by xylazine hydrochloride (Rompun ${ }^{\mathrm{R}}$; Bayer, Leverkusen, Germany; $5 \mathrm{mg} / \mathrm{kg}$ ). The mid-cervical trachea was exposed and a Hamilton syringe was introduced through a small incision between two tracheal cartilages and forwarded to the right main stem bronchus, and $1 \mathrm{ml}$ of the fluorescent tracer Fast-Blue (Dr. Illing, Gross-Umstadt, Germany; 2\% aqueous solution containing $1 \%$ dimethylsulphoxide) were injected into the right hilar region. Animals were allowed to recover until skin test and allergen challenge as described before. Tissues (injection sites and nodose ganglia) were removed $1 \mathrm{~d}$ after challenge and treated as described for double-labeling immunohistochemistry.

Radioimmunoassay. Samples of the upper thoracic trachea and of peripheral lung were snap frozen in liquid nitrogen. Radioimmunoassay for SP, NKA, and CGRP was performed as described before (27). The following antibodies were checked for cross-reactivities and used in this study: anti-SP-antibody Rd2 (donated by Dr. S. Leeman, University of Boston, Boston, MA), anti-NKA-antibody K12 (donated by Dr. E. Theodorsson, Karolinska Hospital, Stockholm, Sweden) and anti-CGRP-antibody RAS6009 (Peninsula Laboratories Inc., St. Helens, UK). Non radioactive synthetic peptides were obtained from Peninsula and radioactively labeled peptides from Amersham.

Statistical analysis of data. The data obtained from radioimmunoassay, in situ hybridization, double-labeling immunohistochemistry, and northern blot analysis were expressed as mean values, with the standard error of the mean (SEM) and compared in sensitized animals between the different time points investigated and between sensitized and control animals by Mann Whitney U-test. In each 
group and at each time point, data from three to five animals were analyzed.

\section{Results}

Active sensitization of guinea pigs to ovalbumin. The sensitization protocol shows very consistent results because no animal $(n=173)$ had to be excluded from the study due to falsepositive or false-negative skin testing. In sensitized animals, a massive flare reaction and more than a doubling of skin thickness is observed, whereas no reaction is seen in control animals.

Scanning electron microscopy. The airways of sensitized animals show the typical signs of an allergic airway inflammation, e.g., epithelial shedding and accumulation of inflammatory cells. These are seen 12 and $24 \mathrm{~h}$ after allergen challenge, and persist $3 \mathrm{~d}$ after challenge (Fig. $1 A$ ), whereas, after $7 \mathrm{~d}$, the respiratory epithelium recovers from the signs of inflammation. In contrast, the respiratory epithelium of control animals

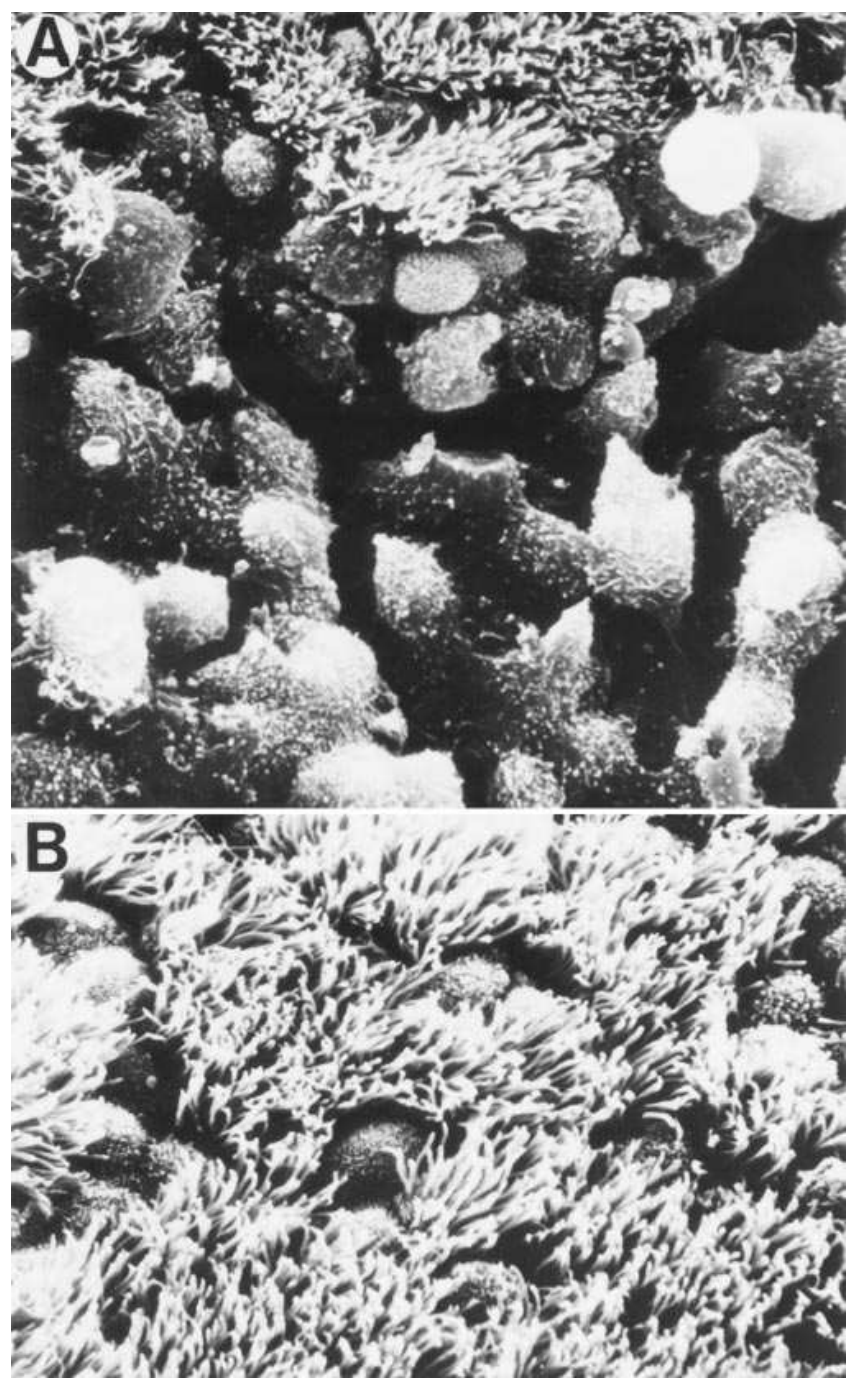

Figure 1. Scanning electron microscopic photomicrograph of the tracheal epithelium $3 \mathrm{~d}$ after inhalative ovalbumin challenge. Signs of an allergic inflammation (e.g., epithelial shedding) are observed in sensitized animals $(A)$, whereas the respiratory epithelium of control animals is intact $(B) . \times 2,500$. is intact at all investigated time points after allergen challenge (Fig. $1 B$ ).

Northern blot analysis. During the allergic airway inflammation, a moderate increase $(\sim 20 \%)$ of PPT-mRNA-/actinmRNA-ratios in the nodose ganglion $12 \mathrm{~h}$ after inhalative allergen challenge is observed, returning to values comparable to prechallenge ratios and followed by a decrease $(\sim 20 \%)$ at days 3 and 7 after challenge (Fig. 2). These changes are statistically significant $(P<0.05)$. In contrast to the nodose ganglion, the PPT-mRNA-/actin-mRNA-ratios obtained for the jugular ganglion did not exhibit marked changes in preliminary exper-
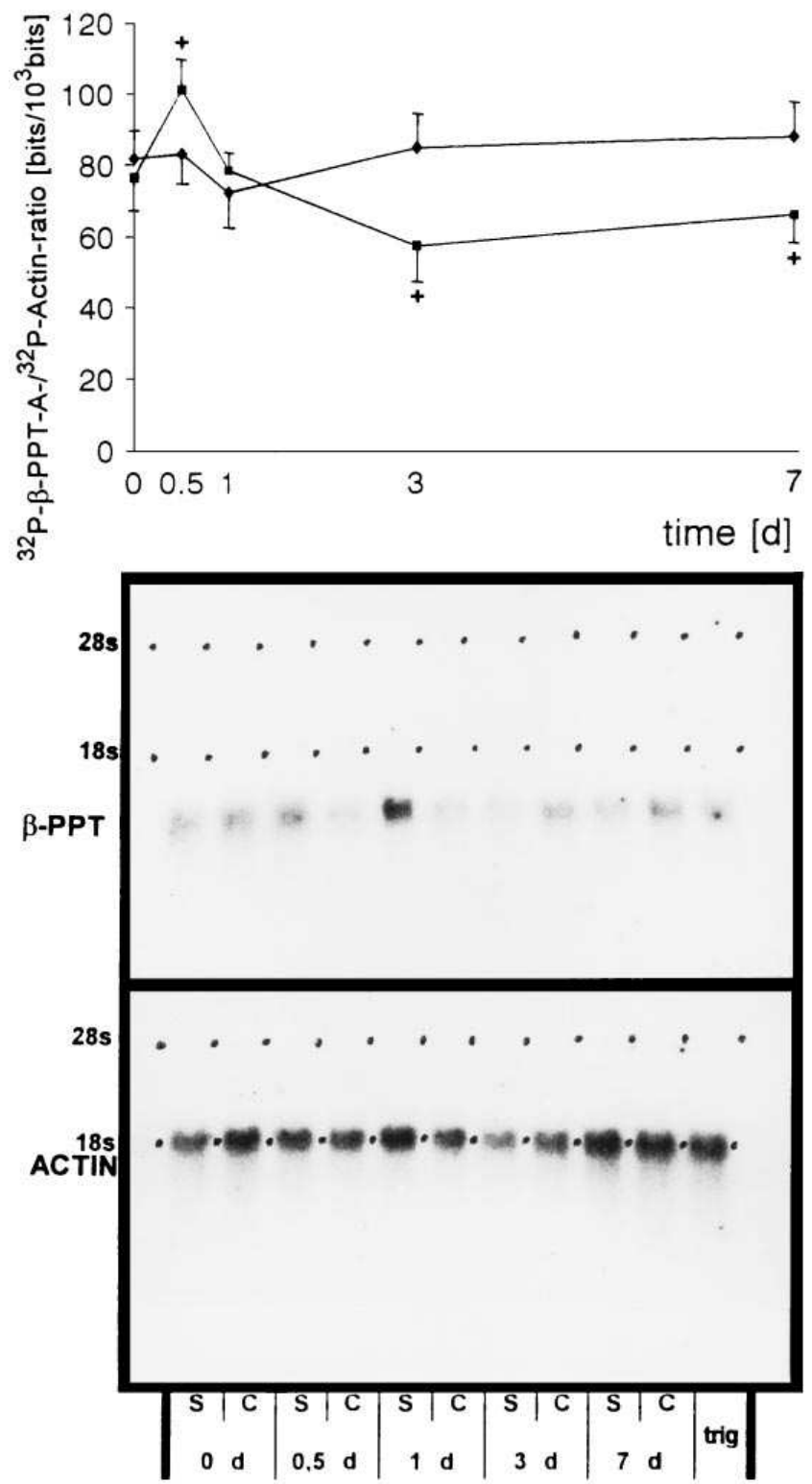

Figure 2. Northern blot analysis of $\beta$-PPT-A mRNA and $\beta$-actin mRNA expression in the guinea pig nodose ganglion. Quantification using a PhosphorImager reveals increased PPT/actin-ratios in sensitized $(S)$ guinea pigs (rectangles) $12 \mathrm{~h}$ after allergen challenge followed by a decrease 3 and $7 \mathrm{~d}$ after challenge as compared to control (C) animals (diamonds). X-ray documentation of the hybridized blots is given in the lower panels. Each value is a mean of three independent experiments \pm SEM. ${ }^{+} P<0.05$; trig $=$ trigeminal ganglion. 

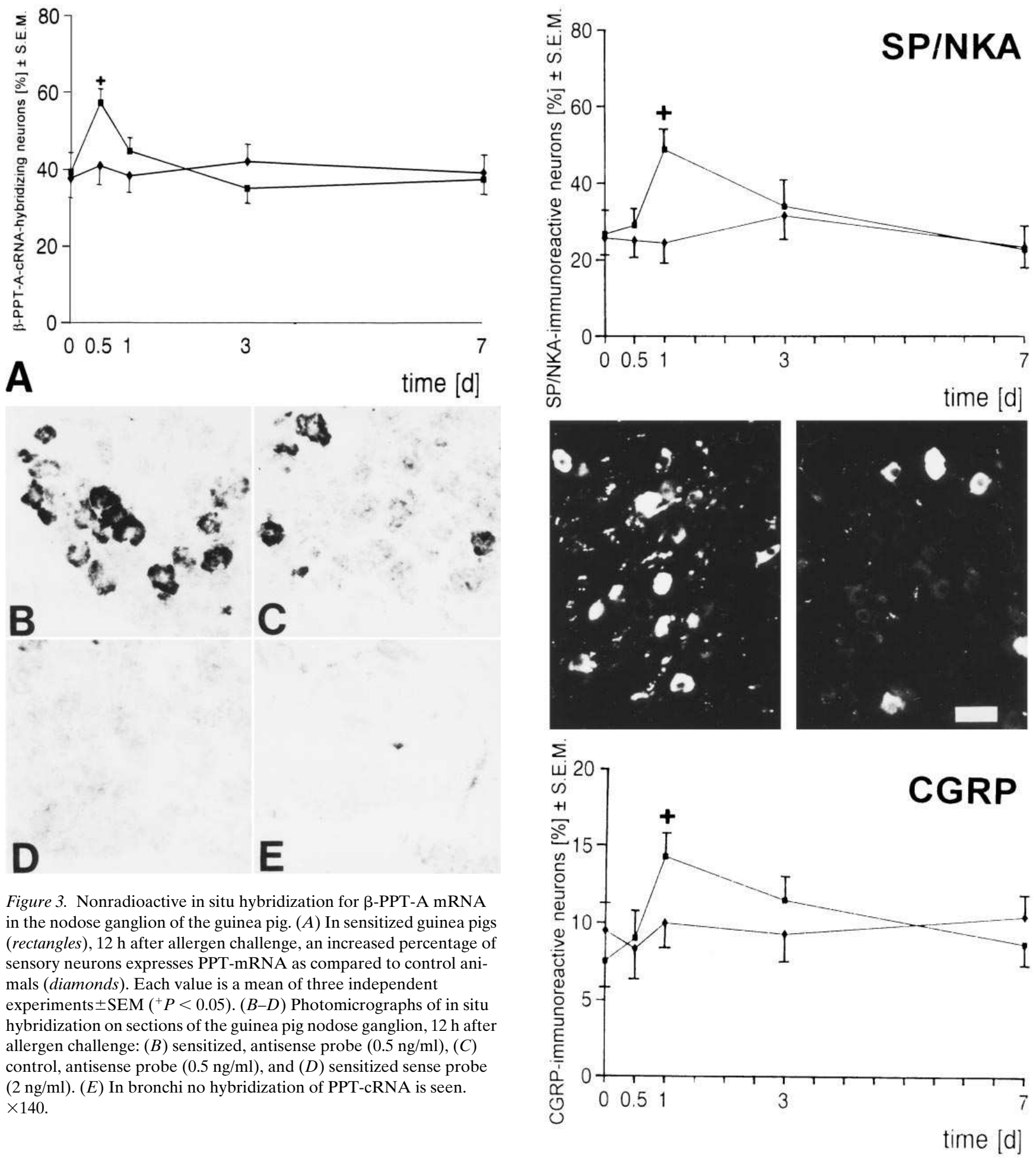

Figure 3. Nonradioactive in situ hybridization for $\beta$-PPT-A mRNA in the nodose ganglion of the guinea pig. $(A)$ In sensitized guinea pigs (rectangles), $12 \mathrm{~h}$ after allergen challenge, an increased percentage of sensory neurons expresses PPT-mRNA as compared to control animals (diamonds). Each value is a mean of three independent experiments \pm SEM $\left({ }^{+} P<0.05\right)$. $(B-D)$ Photomicrographs of in situ hybridization on sections of the guinea pig nodose ganglion, $12 \mathrm{~h}$ after allergen challenge: $(B)$ sensitized, antisense probe $(0.5 \mathrm{ng} / \mathrm{ml}),(C)$ control, antisense probe $(0.5 \mathrm{ng} / \mathrm{ml})$, and $(D)$ sensitized sense probe $(2 \mathrm{ng} / \mathrm{ml})$. (E) In bronchi no hybridization of PPT-cRNA is seen. $\times 140$.

iments. So, the investigation of these ganglia was not repeated and insufficient data for statistical analysis was obtained. In the lung, no PPT-mRNA is detectable at any time point in tissues from neither control nor sensitized animals.

In situ hybridization. The number of neurons in the nodose ganglion expressing PPT-mRNA increases $12 \mathrm{~h}$ after allergen challenge (Fig. $3 A$ and $B$ ) by one-third as compared to sensitized animals investigated at other time points after allergen challenge and to control groups $(P<0.025$, Fig. $3 A$ and $C)$. Incubation of the ganglia with $2 \mathrm{ng} / \mathrm{ml}$ of the sense probe

Figure 4. Immunohistochemistry for SP/NKA and CGRP in the nodose ganglion reveals an increase of immunoreactive perikarya in sensitized (rectangles) as compared to control (diamonds) guinea pigs $24 \mathrm{~h}$ after allergen challenge, returning to control values 3 and 7 days after challenge. Each value is a mean of five independent experiments \pm SEM $\left({ }^{+} P<0.025\right)$. Photomicrographs of SP-/NKAimmunoreactive neurons in the nodose ganglion $24 \mathrm{~h}$ after allergen challenge. In sensitized animals, a higher percentage of neurons is immunoreactive for SP/NKA as compared to control animals. Scale bar: $50 \mu \mathrm{m}$. 

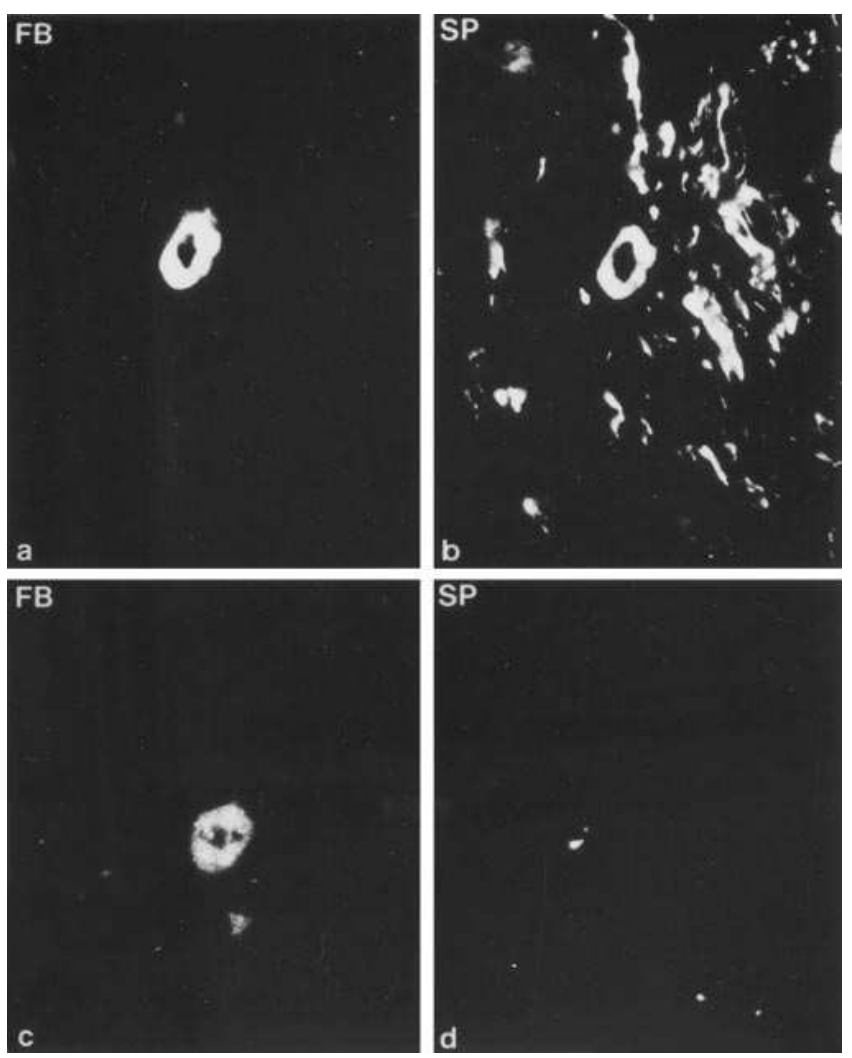

Figure 5. Retrograde neuronal tracing combined with immunohistochemistry for SP/NKA. ( $a$ and $b$ ) A sensory neuron in the guinea pig nodose ganglion, retrogradely labeled from the lung hilum with the fluorescent tracer Fast-Blue $(a)$ and killed $24 \mathrm{~h}$ after allergen challenge, displaying strong immunoreactivity for SP/NKA $(b)$. $(c$ and $d$ ) Retrogradely labeled neuron in the nodose ganglion of a control animal $(c)$ is negative in immunohistochemistry for SP/NKA. $\times 160$.

resulted in absence of labeling (Fig. $3 \mathrm{D}$ ). In the airways, no hybridization of PPT-cRNA is seen in sensitized and control animals (Fig. 3 E).

Immunohistochemistry. The percentage of SP/NKA-immunoreactive neurons in the nodose ganglion increases from $25 \%$ to a maximum of $48 \% 24 \mathrm{~h}$ following allergen challenge and returned to prechallenge values $3 \mathrm{~d}$ after allergen challenge (Fig. $4 A-C$ ).

Similarly, but to a lesser extent, the percentage of CGRPimmunoreactive neurons rises from $8 \%$ to a maximum of $15 \%$ $24 \mathrm{~h}$ after allergen challenge (Fig. $4 \mathrm{D}$ ). These increases are statistically significant $(P<0.025)$.

Retrograde neuronal tracing. In the six nodose ganglia of three control animals an overall of 1,261 neurons was found to be retrogradely labeled from the right lung hilum (Fig. $5 \mathrm{C}$ and $D$, left and right). Only three of them are immunoreactive for SP/NKA, which corresponds to $0.2 \%$. In the nodose ganglia of sensitized and challenged guinea pigs 938 retrogradely FastBlue-labeled neurons were identified $24 \mathrm{~h}$ after allergen challenge. $98(10.4 \%)$ of these neurons exhibited SP/NKA immunoreactivity (Fig. $5 A$ and $B$ ).

Radioimmunoassay. Neuropeptide concentrations in the trachea are higher than in the lung and show a large inter individual variation. However, no significant differences between trachea

lung

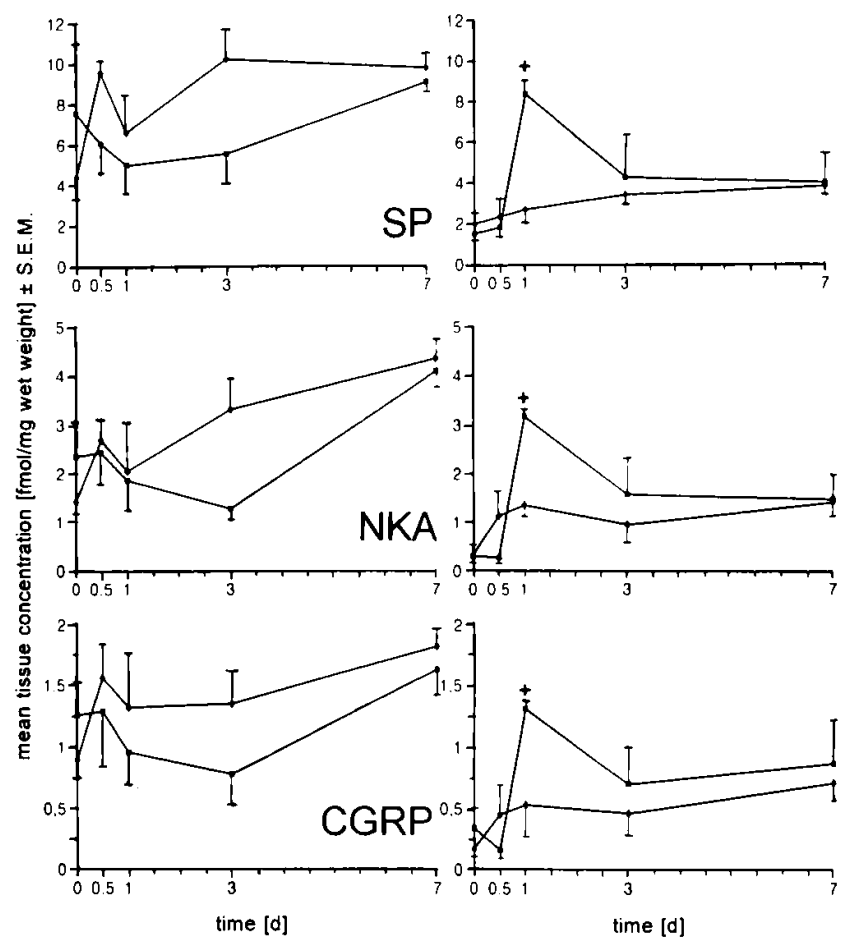

Figure 6. Neuropeptide levels in the airways as determined by radioimmunoassay. SP, NKA, and CGRP levels/mg tissue wet weight are higher in trachea than in lung tissues but show a large variation without significant changes at the time points investigated. In contrast, in lung tissues the neuropeptide levels show smaller variations, and a statistically significant $\left({ }^{+} P<0.01\right)$ three- to fourfold increase is observed in tissues of sensitized guinea pigs (rectangles) $24 \mathrm{~h}$ after allergen challenge as compared to other investigated time points and to control animals (diamonds).

sensitized and control animals before or during the course of the allergic airway inflammation are apparent (Fig. 6). In contrast, in lung tissues the concentrations of SP, NKA, and CGRP show less variation. During the time course of the allergic inflammation, three- to fourfold increases of the tissue concentrations of SP, NKA, and CGRP are observed $24 \mathrm{~h}$ after inhalative ovalbumin challenge, returning to control values at days 3 and 7 after challenge (Fig. 6). These increases are statistically significant $(P<0.01)$ as compared to the other time points investigated and to control animals.

\section{Discussion}

The present study provides evidence for plasticity of the expression of PPT-mRNA, tachykinin peptides, and CGRP in the nodose ganglion of the guinea pig induced by allergic airway inflammation. To our knowledge, induction of plastic changes in vagal sensory neurons of the nodose ganglion by inflammation have not, so far, been reported. Changes in the biosynthesis of sensory neuropeptides have been found in several animal models of tissue inflammation or of nerve damage. For example, the number of PPT-mRNA expressing neurons in rat dorsal root ganglia (DRG) increases after formalin injection into the hindlimb (20). Additionally, enhanced biosynthe- 
sis and axonal transport of neuropeptides in the sensory neurons was shown in a model of adjuvant-induced arthritis (21). In inflammatory diseases of the viscera, the involvement of tachykinin signals has been inferred by the demonstration of certain trophic changes. For example, neurokinin 1 (NK1) receptors are upregulated in human inflammatory bowel diseases, ulcerative colitis, and Crohn's disease (28) as well as in human asthma (29). In inflammatory bowel disease, it has been shown that inflammatory cells, e.g., mast cells are sources of tachykinins (28). In this model of allergic airway inflammation, no evidence of PPT-mRNA or tachykinin neuropeptide expression was seen in inflammatory cells in in situ hybridization and immunohistochemistry of lung sections. Since mast cells are also important sources of degrading enzymes and targets of tachykinins, indirect effects may influence the inflammatory reaction. In this respect, it also seems likely that substances derived from the inflamed tissue are responsible for inducing the trophic changes in tachykinin and tachykinin-receptor metabolism. The importance of target-derived factors for regulating the biosynthesis of the tachykinins and other neuropeptides is also evident from the decreased PPT-mRNA and CGRP-mRNA levels in sensory neurons observed following axotomy $(30,31)$. Indeed, the regulation of PPT-gene expression in dorsal root ganglion sensory neurons is dependent on several factors (32), including nerve growth factor (NGF) and IL-1, and involves AP1- and AP2-transcription factors (33). However, it remains to be determined whether neurotrophic factors, or other mechanisms such as changes in the neuronal electrical activity, are responsible for the rapid and transient induction of PPT-mRNA expression in the nodose ganglion that has been demonstrated here in response to allergen challenge.

The active sensitization of guinea pigs to ovalbumin is a commonly used model for allergic airway diseases. Its reliability and reproducibility was evident in this study because no animal had to be excluded from the study due to negative skin testing. Although this model may not entirely reflect the situation in human allergic asthma, many similarities are observed: histological features (34), allergen-induced eosinophilia and hyperreactivity (22), and early- and late-phase airway obstruction after allergen challenge (35).

A moderate, but statistically significant increase of the relative levels of PPT-mRNA is observed $12 \mathrm{~h}$ after allergen challenge, followed by a decrease after between 3 and $7 \mathrm{~d}$. This increase is due to de novo induction of PPT-mRNA-expression in nodose ganglion primary afferent neurons. In the rat model of adjuvant-induced arthritis, similar increases of PPT- and CGRP-mRNA in dorsal root ganglion sensory neurons, $8 \mathrm{~h}$ after induction of inflammation, have been reported (36).

As observed in earlier reports of combined in situ hybridization and immunohistochemistry, a higher percentage of PPT expressing sensory neurons was detected, in the present study, using in situ hybridization (35-40\%) than using immunohistochemistry $(\sim 25 \%)$. But, with the induction of PPT expression, the changes observed by in situ hybridization paralleled those observed by immunohistochemistry which were, however, later due, presumably, to translation and posttranslational events. The experiments of Huang and colleagues (31), in contrast, revealed changes in CGRP expression in rat nodose ganglion sensory neurons after axotomy using in situ hybridization, that were not apparent using immunohistochemistry (37). In rat nodose ganglion sensory neurons, NPY-gene expression was apparent from in situ hybridization analysis, whereas NPY immunoreactivity was found only occasionally (38). These discrepancies may reflect differences in the relative sensitivities of the different methods or indicate differential regulation of posttranscriptional and posttranslational processes.

$24 \mathrm{~h}$ after allergen challenge of sensitized guinea pig, an increased number of sensory neurons is immunoreactive for SP/ NKA. However, because a model of systemic sensitization and allergen challenge was used, the increase in tachykinin immunoreactivity cannot be assumed to be caused by airway inflammation. Therefore, using retrograde tracing, the projections of those sensory neurons of the nodose ganglion, in which tachykinin immunoreactivity was induced by sensitization and inhalative allergen challenge, were identified.

In normal guinea pigs, nodose ganglion sensory neurons are the major source of the afferent innervation of the airways (17), but are not immunoreactive for substance $\mathrm{P}$ and neuroki$\operatorname{nin}$ A. However, the results of our tracing experiments suggest that the vast majority of the increased number of tachykinin synthesizing cells that occurs in the nodose ganglion following allergen challenge can be accounted for by the induction of tachykinin biosynthesis in neurones projecting to the airways. But, allergic reactions in other organs supplied by the nodose ganglion, such as the upper gastrointestinal tract, may also contribute to the induction of tachykinin biosynthesis. The rapid increase of tachykinin immunoreactivity with a similarly rapid fall to control values parallels, with a delay of $12 \mathrm{~h}$, the increase and decrease of PPT-mRNA in these neurons. Whereas in other models of chronic inflammation, such as the adjuvant-induced paw inflammation, a long lasting increase of the percentage of immunoreactive sensory neurons was reported (39). The acute airway inflammation, with a single allergen challenge, is associated with a shorter period of increased neuropeptide content in the perikarya.

Changes in CGRP immunoreactivity are similar to the changes in tachykinin immunoreactivity but are less marked. In the course of airway inflammation, immunoreactive CGRP is induced in nodose ganglion sensory neurons in parallel to the changes observed for SP and NKA.

Decreased levels of SP and NKA have been reported in lung tissue extracts during the early phase following allergen challenge (40), indicating a release of neuropeptides stored in sensory nerve endings. In contrast, three- to fourfold elevated levels of the sensory neuropeptides were found in this study in lung tissue extracts of sensitized animals $24 \mathrm{~h}$ after allergen challenge, concomitant to the increase of tachykinin-immunoreactive neurons innervating the airways. These increased levels in the lung tissues $24 \mathrm{~h}$ after allergen challenge are apparently due to an increased biosynthesis of the neuropeptides in the ganglion and an increased transport to the peripheral ending. Similarly, in the model of the adjuvant-induced paw inflammation a rapid and increased axonal transport of sensory neuropeptides towards the peripheral terminals was demonstrated (21).

In our animal model, the apparent increased delivery of tachykinins to the airways, which is concomitant with their increased biosynthesis, is consistant with the elevated levels of tachykinins found in broncho-alveolar lavage fluids of asthmatic patients compared to those of healthy volunteers (7). The measurement of the release of the bioactive at present is only possible in functional studies using specific receptor an- 
tagonists. In the present study, we have addressed changes of neuropeptide biosynthesis and chosen to measure the tissue concentrations of neuropeptides. Hence, also contributing to the increased tachykinin content of the airways following allergen challenge may be decreased postsecretory breakdown of peptide. A loss of extracellular peptidase activity has been reported to occur in the inflamed airways (41).

As discussed earlier, the model used in the present study shows many similarities to the human asthma. One of the important parallels to human asthma is the early and late onset airway obstruction (35), due to an increased airway reactivity in the early phase after allergen challenge, which, following a period of several hours of attenuation, increases again in the late phase of the allergic reaction, $24 \mathrm{~h}$ after allergen challenge (22). In the early phase of the allergic reaction, release of sensory neuropeptides as described by Yoshihara (40) may account for the enhanced nonspecific airway hyperresponsiveness (8). The data presented in this study indicate that the further augmented airway hyperresponsiveness during the late response, $24 \mathrm{~h}$ after allergen challenge, is very closely associated with an induction of biosynthesis of sensory neuropeptides in the nodose ganglion and an enhanced transport to the peripheral nerve endings. Because these sensory neuropeptides may mediate neurogenic inflammation, these plastic changes in the airway innervation could be a crucial pathophysiological process.

\section{Acknowledgments}

We thank Prof. K. Tiedemann for the assistance with the scanning electronmicroscopical imaging and Ms. Birgit Hoba, Mrs. C. MerteGrebe, Mrs. T. Seitz, and Mrs. A.C. Saria for their expert technical assistance.

This study was supported by the DFG (Forschergruppe Neuronale Plastizität Zi 110/22-1), the P.E. Kempkes-Stiftung, Marburg (grant to G. McGregor) and the Austrian Science Foundation (F206 to A. Saria).

\section{References}

1. Barnes, P.J., J.N. Baraniuk, and M.G. Belvisi. 1991. Neuropeptides in the respiratory tract. Part I. Am. Rev. Respir. Dis. 144:1187-1198.

2. Barnes, P.J., J.N. Baraniuk, and M.G. Belvisi. 1991. Neuropeptides in the respiratory tract. Part II. Am. Rev. Respir. Dis. 144:1391-1399.

3. Saria, A., C.R. Martling, Z. Yan, E. Theodorsson-Norheim, R. Gamse, and J.M. Lundberg. 1988. Release of multiple tachykinins from capsaicin sensitive sensory nerves in the lung by bradykinin, histamine, dimethylphenyl piperazonium, and vagal nerve stimulation. Am. Rev. Respir. Dis. 137:1330-1335.

4. Solway, J., and A.R. Leff. 1991. Sensory nerves and airway function. $J$. Appl. Physiol. 71:2077-2087.

5. Pernow, B. 1985. Role of tachykinins in neurogenic inflammation. J. Immunol. 135 (Suppl.):812S-815S.

6. Barnes, P.J. 1990. Neurogenic inflammation in airways and its modulation. Arch. Int. Pharmacodyn. Ther. 303:67-82.

7. Nieber, K., C.R. Baumgarten, R. Rathsack, J. Furkert, P. Oehme, and G. Kunkel. 1992. Substance-P and beta-endorphin-like immunoreactivity in lavage fluids of subjects with and without allergic asthma. J. Allergy Clin. Immunol. 90: 646-652.

8. Hsiue, T.R., A. Garland, D.W. Ray, M.B. Hershenson, A.R. Leff, and J. Solway. 1992. Endogenous sensory neuropeptide release enhances nonspecific airway responsiveness in guinea pigs. Am. Rev. Respir. Dis. 146:148-153.

9. Escher, E., R. Couture, C. Poulos, N. Pinas, J. Mizrahi, D. Theodoropoulos, and D. Regoli. 1982. Structure-activity studies on the C-terminal amide of substance P. J. Med. Chem. 25:1317-1321.

10. Frossard, N., and C. Advenier. 1991. Tachykinin receptors and the airways. Life Sci. 49:1941-1953.

11. Lei, Y.H., P.J. Barnes, and D.F. Rogers. 1992. Inhibition of neurogenic plasma exudation in guinea pig airways by CP-96.345, a new non-peptide NK1 receptor antagonist. Br. J. Pharmacol. 105:261-262.

12. Kuo, H.P., J.A. Rohde, K. Tokuyama, P.J. Barnes, and D.F. Rogers.
1990. Capsaicin and sensory neuropeptide stimulation of goblet cell secretion in guinea pig trachea. J. Physiol. 431:629-641.

13. Lou, Y.P., L.Y. Lee, H. Satoh, and J.M. Lundberg. 1992. Inhibitory effects of capsazepine and the NK2 antagonist SR 48.968 on bronchconstriction evoked by sensory nerve stimulation in guinea pigs. Br. J. Pharmacol. 109:765773.

14. Martling, C.R., A. Saria, J.A. Fischer, T. Hökfelt, and J.M. Lundberg. 1988. Calcitonin gene-related peptide and the lung: neuronal coexistence with substance P, release by capsaicin and vasodilatory effect. Regul. Pept. 20:125139

15. Lundberg, J.M., T. Hökfelt, C.R. Martling, A. Saria, and C. Cuello. 1984. Substance-P immunoreactive sensory nerves in the lower respiratory tract of various mammals including man. Cell Tissue Res. 235:251-261.

16. Springall, D.R., A. Cadieux, H. Oliveira, H. Su, D. Royston, and J.M. Polak. 1987. Retrograde tracing shows that CGRP-immunoreactive nerves of rat trachea and lung originate from vagal and dorsal root ganglia. J. Auton. Nerv. Syst. 20:155-166.

17. Kummer, W., A. Fischer, R. Kurkowski, and C. Heym. 1992. The sensory and sympathetic innervation of guinea pig lung and trachea as studied by retrograde neuronal tracing and double-labeling immunohistochemistry. Neuroscience. 49:715-737.

18. Saria, A., C.R. Martling, C.J. Dalsgaard, and J.M. Lundberg. 1985. Evidence for substance P-immunoreactive spinal afferents that mediate bronchoconstriction. Acta Physiol. Scand. 125:407-414.

19. Hökfelt, T., X. Zhang, and Z. Wiesenfeld-Hallin. 1994. Messenger plasticity in primary sensory neurons following axotomy and its functional implications. Trends Neurosci. 7:22-30.

20. Noguchi, K., Y. Morita, H. Kiyama, K. Ono, and M. Tohyama. 1988. A noxious stimulus induces the preprotachykinin-A gene expression in the rat dorsal root ganglion cells following peripheral axotomy. Brain Res. 464:31-35.

21. Donnerer, J., R. Schuligoi, and C. Stein. 1992. Increased content and transport of substance P and calcitonin gene-related peptide in sensory nerves innervating inflamed tissue evidence for a regulatory function of nerve growth factor in vivo. Neuroscience. 49:693-698.

22. Sanjar, S., S. Aoki, A. Kristersson, D. Smith, and J. Morley. 1990. Antigen challenge induces pulmonary airway eosinophil accumulation and airway hyperreactivity in sensitized guinea pigs: the effect of anti-asthma drugs. $B r . J$. Pharmacol. 99:679-686.

23. Chirgwin, J.M., A.E. Przybyla, R.J. MacDonald, and W.J. Rutter. 1979. Isolation of biologically active ribonucleic acid from sources enriched in ribonuclease. Biochemistry. 18:5294-5298.

24. McGregor, G.P., R. Hartel, R. Haberberger, W. Kummer, and K.H. Voigt. 1995. Preprotachykinin-A gene expression occurs transiently in the developping rat endocrine pancreas and can be regulated in RIN-m5F cells. Endocrinology. 136:2538-2546.

25. Krause, J.E., J.M. Chirgwin, M.S. Carter, Z.S. Xu, and A.D. Hershey. 1987. Three rat preprotachykinin in RNAs encode the neuropeptides substance $\mathrm{P}$ and neurokinin A. Proc. Natl. Acad. Sci. USA. 84:881-885.

26. Mundel, P., S. Bachmann, M. Bader, A. Fischer, W. Kummer, B. Mayer, and W. Kriz. 1992. Expression of nitric oxide synthase in kidney macula densa cells. Kidney Int. 42:1017-1019.

27. Humpel, C., C. Neudorfer, W. Philipp, H.J. Steiner, C. Haring, K.W. Schmid, J. Schwitzer, and A. Saria. 1992. Effects of bright certificial light on monoamines and neuropeptides in eight different brain regions compared in a pigmented and nonpigmented rat strain. J. Neurosci. Res. 32:605-612.

28. Mantyh, P.W., and J.E. Maggio. 1992. Substance P and the response to tissue injury. Regul. Pept. (Suppl. 1):S19.

29. Adcock, I.M., M. Peters, C. Gelder, H. Shirasaki, C.R. Brown, and P.J. Barnes. 1993. Increased tachykinin receptor gene expression in asthmatic lung and its modulation by steroids. J. Mol. Endocrinol. 11:1-7.

30. Noguchi, K., E. Senba, Y. Morita, M. Sato, and M. Tohyama. 1989. Prepro-VIP and pre-pro-tachykinin mRNAs in the rat dorsal root ganglion cells following peripheral axotomy. Mol. Brain Res. 6:327-330.

31. Huang, F.L., H. Zhuo, C. Sinclair, M.E. Goldstein, J.T. McCabe, and C.J. Helke. 1994. Peripheral deafferentation alters calcitonin gene-related peptide in RNA expression in visceral sensory neurons of the nodose and petrosal ganglia. Mol. Brain Res. 22:290-298.

32. Levine, J.D., H.L. Fields, and A.I. Basbaum. 1993. Peptides and the primary afferent nociceptor. J. Neurosci. 13:2273-2286.

33. Donaldson, L.F., D.S. McQueen, and J.R. Seckl. 1994. J. Physiol. 475: 139P (Abstr.)

34. Laitinen, L.A., M. Heino, A. Laitinen, T. Kava, and T. Haahtela. 1985. Damage of the airway epithelium and bronchial reactivity in patients with asthma. Am. Rev. Respir. Dis. 131:599-606.

35. Hutson, P.A., M.K. Church, T.P. Clay, P. Miller, and S.T. Holgate. 1988. Early and late-phase bronchoconstriction after allergen challenge on nonanesthetized guinea pigs. I. The associations of disordered airway physiology to leukocyte infiltration. Am. Rev. Respir. Dis. 137:548-557.

36. Donaldson, L.F., A.J. Harmar, D.S. McQueen, and J.R. Seckl. 1992. Increased expression of preprotachykinin, calcitonin gene-related peptide, but not vasoactive intestinal peptide messenger RNA in dorsal root ganglia during the development of adjuvant monoarthritis in the rat. Mol. Brain Res. 16:143149. 
37. Helke, C.J., and A. Rabchevsky. 1991. Axotomy alters putative neurotransmitters in visceral sensory neurons of the nodose and petrosal ganglia. Brain Res. 551:44-51.

38. Kummer, W., S. Bachmann, W.L. Neuhuber, J. Hänze, and R.E. Lang. 1993. Tyrosinehydroxylase-containing vagal afferent neurons in the rat nodose ganglia are independent from neuropeptide $\mathrm{Y}$ containing populations and project to esophagus and stomach. Cell Tissue Res. 271:135-144.

39. Donnerer, J., R. Schuligoi, C. Stein, and R. Amann. 1993. Upregulation release and axonal transport of substance $\mathrm{P}$ and calcitonin gene-related peptide in adjuvant inflammation and regulatory function of nerve growth factor Regul. Pept. 46:150-154.

40. Yoshihara, S. 1988. Immunoreactive substance P levels in respiratory tract tissues of normal and airway allergic guinea-pigs. Jpn. J. Allergol. 37:11611168.

41. Nadel, J.A. 1992. Neurogenic inflammation in the airways and its modulation by peptidases. Ann. NY Acad. Sci. 664:408-414. 